\title{
Novel In-Service Combustion Instability Detection Using the Chirp Fourier Higher Order Spectra
}

\author{
Len Gelman ${ }^{1}$, Colin Parrish ${ }^{2}$, Ivan Petrunin ${ }^{3}$ and Mark Walters ${ }^{4}$ \\ ${ }^{1,3}$ Cranfield University, Cranfield, Bedfordshire, MK43 OAL, UK \\ l.gelman@cranfield.ac.uk \\ i.petrunin@cranfield.ac.uk \\ ${ }^{2,4}$ Rolls-Royce, Derby, Derbyshire, DE24 8BJ, UK \\ mark.walters@rolls-royce.com \\ colin.parrish@rolls-royce.com
}

\begin{abstract}
Combustion instabilities, known as "rumble" and "screech" are the self-excited aerodynamic instabilities in the gas turbine combustor. They cause the premature failures of the gas turbine components, and, consequently, the failure of the gas turbine as a whole. Because of the complex physical effects underlying the rumble and the screech phenomena, it is difficult to eliminate them completely at the design stage. Therefore, special attention should be paid to the detection of the combustion instabilities in the gas turbine in order to prevent its prolonged operation in this mode. There are known techniques, which are able to detect the rumble and the screech in gas turbines. Most of them do not consider the combustion instabilities as non-linear and non-stationary events and, therefore, have lower detection efficiency. Novel technique for in-service combustion instability detection is implemented in this paper. This technique overcomes the limitations of the existing solutions.
\end{abstract}

\section{INTRODUCTION}

The combustion instabilities can be caused by multiple reasons. According to Williams (1985) the instabilities in combustion can be classified based on the size of the components involved in the instability formation: intrinsic (local and short distance interactions in the flow; high frequency of the effect), chamber (medium size instabilities, such as coupling of vortices in the flow with the flame front; medium frequencies) and system (whole combustor is

Len Gelman et al. This is an open-access article distributed under the terms of the Creative Commons Attribution 3.0 United States License, which permits unrestricted use, distribution, and reproduction in any medium, provided the original author and source are credited. involved, resulting in low frequency effects). It is common (Ebrahimi, 2006, Wroblewski \& Grabill, 2001) to use term "rumble" for description of the low frequency instabilities in the range $50-120 \mathrm{~Hz}$ and term "screech" for a higher frequency oscillations in the range $120-600 \mathrm{~Hz}$. Later in this work the term "rumble" will be used to describe both types of combustion instabilities in order to preserve the clarity of the material presentation.

The instabilities in the combustion process cause the pressure variations in the combustion chamber in the form of acoustic waves. Due to multiple resonances (both acoustical and mechanical) in the combustor and surrounding structures the initially strong effect can be further amplified, supporting itself and reaching the levels, where the accelerated fatigue and degradation of the components lead to the failure. For example, it is mentioned by Wroblewski \& Grabill (2001) that in near field the intensity of acoustic tones created by combustion instabilities, e.g. rumble, can reach $160 \mathrm{~dB}$. At these sound pressure levels rumble can cause fatigue in structures as well as other vibration related problems. In addition, the combustion instabilities are considered now as the significant contributors to the overall turbine noise in view of the progress in reduction of noise from other sources in gas turbines (Dowling \& Mahmoudi, 2015).

Despite the efforts made at the design stage to prevent the appearance of the combustion instabilities at stationary operational regimes of the turbine it is still difficult to predict and eliminate them at every turbine regime, especially at nonstationary ones, associated with changes in the shaft speed (Yao, Gao, Zhu, Dowling \& Bray, 2012). Therefore, the rumble detector must be present in the gas turbine monitoring system, allowing for elimination of the instabilities, for instance, by variation of the fuel supply (Lubarsky \& 
Yeshayahou, 1998, Richman \& Richman, 2000, Hermann, Orthmann, Hoffman \& Berenbrink, 2000) and preventing thus the turbine failures.

Considering the mentioned above features of the rumble the combustion instabilities can be characterized as a very complex, non-linear and non-stationary effects, which require application of the corresponding signal processing techniques for the effective in-service detection.

Another challenge in the combustion instability detection is associated with the growing interest to the integrated turbine health monitoring systems (Grabill, Seale, Wroblewski \& Brotherton, 2002) able to perform the monitoring of the turbine condition using the minimum number of sensors, preferably accelerometers, as this provides cost effective, flexible and more reliable solution in comparison to use of the pressure transducers, especially high temperature ones. In such systems positions of the accelerometers are rarely optimal for a particular monitoring task; therefore, the questions of optimal signal processing and efficient detection of the combustion instabilities become very important.

Therefore, the purposes of this paper are

- to investigate and develop in-service combustion instability detection by signal processing techniques suitable for both non-linear and non-stationary phenomena using the casing accelerometer signal, significantly contaminated by multiple interfering components generated by the gas turbine during its operation.

- to compare the proposed techniques.

\section{STATE OF THE ART}

Due to high amplitudes of the acoustic tones generated by the combustion instabilities, even simple detection techniques in time or frequency domain may work well, providing that these events are stationary and the signal is picked by the pressure transducer located close to the place of the rumble occurrence. These techniques are usually based on the comparison of a root mean square (RMS) signal amplitude after the bandpass filtering in the time domain or magnitude of the spectral components in the selected frequency range (using a power spectral density estimate) with a chosen rumble threshold (patent US 3540262, 1970, Yu, Wilson \& Schadow, 1998, Hermann et al., 2000, patent EU EP 2199680A1, 2010, Hochgreb, Dennis, Ayranci, Bainbridge \& Cant, 2013, Motheau, Nicoud \& Poinsot, 2014, Livebardon, Moreau, Gicquel \& Poinsot, 2016). This kind of techniques require stationary input data for the best efficiency and are blind to the non-linearity associated with the rumble phenomenon.

The rumble detection based on processing of the vibration signals taken from accelerometers is significantly more challenging task. It was shown in works of Wroblewski \& Grabill, (2001) and Grabill et al. 2002 that rumble signatures are hardly visible when processing the accelerometer data, while being very clear in the pressure transducer data. This implies the use of more advanced signal processing techniques based on the vibration signal analysis by the short time Fourier transform (STFT). The analysis for the technique proposed by Wroblewski \& Grabill, 2001, Grabill et al. 2002, is performed in the selected frequency range with the following anomaly detection procedure based on the Principal Component Analysis (PCA). Such technique is more suitable for both stationary and non-stationary rumble events due to better suitability of the STFT for analysis of non-stationary signals. This approach is more suitable for detection of non-stationary rumble effects in comparison to simplistic techniques shown in patents EU EP 2199680A1 (2010), US 3540262 (1970), and implemented in works of Wilson \& Schadow (1998), Hermann et al. (2000), Hochgreb et al. (2013), Motheau et al. (2014), Livebardon, et al. (2016). At the same time, this technique is the low-order spectral technique; therefore, it does not allow for detection and recognition of non-linear phenomena, associated with the rumble occurrence. Wroblewski and Grabill (2001) say that their technique still requires data features "that do not change significantly for measurements performed at different operating regimes", meaning effectively the stationarity requirement for the input data.

Requirement of the stationarity is not satisfied for many real world scenarios of the gas turbine operation. Nonstationarity, together with the non-linearity, resulting from excessive acoustic and vibration levels produced by the combustion instabilities and generally non-linear response from the resonating components of the gas turbine, represent significant challenges for existing rumble detection techniques. Therefore, signal processing techniques adapted for the non-linear and non-stationary nature of the rumble phenomena should improve the efficiency of the rumble detection in many practical cases. There are known transforms, which can provide better tracking of the nonstationary rumble components in comparison to the STFT: the short time chirp Fourier transform (Gelman \& Ottley, 2006) and chirp-Wigner transform (Gelman \& Gould, 2007). These transforms can be used for the higher order spectra creation, as it was shown by Gelman and Petrunin (2007), Gelman and Petrunin (2010) and Gelman, Petrunin \& Komoda (2010). These techniques provide the higher sensitivity to the signal non-linearity due to the properties of the higher order spectra.

As the result of the literature search it was found that known rumble detection techniques are not based on the signal processing techniques suitable for essentially non-stationary and non-linear signals. The technique implemented here is the higher order spectra (HOS) (Gelman \& Petrunin, 2007, Gelman \& Petrunin, 2010) based on the chirp Fourier transform (Gelman \& Ottley, 2006). It is suitable for analysis of non-stationary signals with significant non-linearity levels 
and, therefore, it should provide the higher efficiency for the rumble detection in real scenarios of gas turbine operation. Following the undertaken literature review, the novelty of this paper is that the rumble is considered as non-stationary and non-linear event for the first time to the best of authors' knowledge and in order to provide the best efficiency of the detection the non-stationary and non-linear techniques: the short time bicoherences based on the Fourier transform and the short time chirp Fourier transform are implemented and their efficiencies are estimated.

\section{THEORETICAL BACKGROUND}

The HOS techniques considered here for the rumble detection are the third order normalized spectra, i.e. the short time bicoherences based on the Fourier transform and the chirp Fourier transform. From these two techniques, only the latter is adapted for the signals with frequency variable in time.

The classical bicoherence based on the Fourier transform, is generally not suitable for signals with the variable frequency. This property of the classical bicoherence is inherited mostly from the properties of the Fourier transform, which is not suitable for non-stationary signals, e.g. signals with the variable frequency and various frequency and amplitude nonstationarities. The bicoherence based on the chirp Fourier transform (Gelman \& Petrunin, 2007, Gelman \& Petrunin, 2010) is suitable for signals with the variable frequency due to the nature of the short time chirp Fourier transform: the short time chirp Fourier transform is adapted for the signals with any non-linear polynomial frequency variation (Gelman \& Ottley, 2006). Both mentioned HOS techniques applied to long signals with the relatively short duration of the event to be detected may not be effective due to the averaging across time. In works of Gelman and Petrunin (2007), Gelman and Petrunin (2010), the short time implementations of both techniques are proposed, which can be used for in-service rumble detection. The efficiency comparison between the techniques performed in this paper illustrates the importance of the adaptation to the varying rumble frequency while preserving the sensitivity to the non-linearity of the rumble. The short time bicoherence based on the Fourier transform (Gelman \& Petrunin, 2007) implemented here for the rumble detection is computed for a number of external windows, shifted along the whole duration of the analyzed signal:

$$
\operatorname{Bic}_{F T n}\left(f_{k}, f_{l}\right)=\frac{\sum_{i} X_{i}\left(f_{k}\right) X_{i}\left(f_{l}\right) X_{i}^{*}\left(f_{k}+f_{l}\right)}{\sqrt{\sum_{i}\left|X_{i}\left(f_{k}\right) X_{i}\left(f_{l}\right)\right|^{2} \sum_{i}\left|X_{i}\left(f_{k}+f_{l}\right)\right|^{2}}}
$$

where $B i c_{F T n}$ is the bicoherence value for the $n$-th external window; $X_{i}\left(f_{k}\right), X_{i}\left(f_{l}\right)$ and $X_{i}\left(f_{k}+f_{l}\right)$ are the Fourier transforms of the data segment $i$ for the spectral components $k, l$ and $k+l$ with frequencies $f_{k}, f_{l}$ and $f_{k}+f_{l}$; $i=1, \ldots, M, M$ is the number of the data segments forming the $n$-th external window and used for the calculation of the single bicoherence value. As the computed $B i_{F T i}$ contains multiple components, the desired component at a frequency location $\left(f_{k}, f_{l}\right)$ is extracted using the shaft frequency estimate averaged within the external window and component numbers $k$ and $l$.

Another technique used here for the rumble detection is the higher order spectral technique for transient signals with any (i.e. linear and non-linear) polynomial variation of the instantaneous frequency: the short time higher order chirp spectra (HOCS) based on the higher order chirp Fourier transform and time-domain windowing technique (Gelman \& Petrunin, 2007, Gelman \& Petrunin, 2010). For the data processing the third order normalized HOCS is selected: the chirp Fourier bicoherence in its short time version for retrieving the variation of the HOCS in time:

$$
=\frac{\sum_{i} X_{k i}\left(f_{k}\right) X_{l i}\left(f_{l}\right) X_{(k+l) i}^{*}\left(f_{k}+f_{l}\right)}{\sqrt{\sum_{i}\left|X_{k i}\left(f_{k}\right) X_{l i}\left(f_{l}\right)\right|^{2} \sum_{i}\left|X_{(k+l) i}\left(f_{k}+f_{l}\right)\right|^{2}}}
$$

where $B i c_{C F T n}$ is the value of the chirp Fourier based bicoherence for $n$-th external window; $X_{k i}\left(f_{k}\right), X_{l i}\left(f_{l}\right)$ and $X_{(k+l) i}\left(f_{k}+f_{l}\right)$ are the chirp Fourier transforms (Gelman \& Ottley, 2006) of the data segment $i$ for the spectral components $k, l, k+l$ at frequencies $f_{k}, f_{l}$ and $f_{k}+f_{l} ; i=1, \ldots, M, M$ is the number of the data segments forming the external window and used for the calculation of the single bicoherence value. Frequencies $f_{k}, f_{l}$ and $f_{k}+f_{l}$, at which the chirp Fourier transform values are extracted should be chosen from the estimated dependency of the event frequency in time $f_{\text {event }}(t)$, taken with coefficients $k, l, k+l$ respectively.

The short time higher order chirp Fourier transform with the adaptation to the spectral component $k$ can be calculated using the expression as follows:

$$
X_{k i}\left(f_{k}\right)=\frac{1}{T} \int_{0}^{T} w(t) x_{i}(t) \exp \left(-j \Omega_{k}(t)\right) d t,
$$

where $x_{i}(t)$ is the vibration data for the segment $i ; T$ is the length of the data segment; $w(t)$ are the weighting window coefficients, $\Omega_{k}(t)$ is the instantaneous phase of the spectral component $k ; \Omega_{k}(t)=\int_{0}^{t} f_{k}(\tau) d \tau, f_{k}(\tau)$ is the time dependency of the frequency for the spectral component $k$ for which the adaptation will be achieved within the transform. 
The phase dependency should be known a priori or measured independently, based, for example, on online frequency estimation from the tachometer signal.

The short time chirp Fourier bicoherence used here is suitable for detection of short rumble events due to the time-domain windowing, where the time resolution of the detection will be limited by the external window size. It is also suitable for signals with arbitrary polynomial frequency variation in time due to the non-traditional transient kernel of the higher order chirp Fourier transform.

\section{EXPERIMENTAL DATA DESCRIPTION}

The experimental data represent the gas turbine run up from the low idle condition to the maximal speed. The signals used for the rumble detection are recorded from the turbine's casing accelerometer, pressure transducer and tachometers.

Data from the casing accelerometer are usually demonstrating a mixture of the multiple components both wideband and narrowband from various sources. Therefore, identification of weak signals in this mixture often becomes complex. In this case, the data from the pressure transducer were used for locating the rumble components and for their frequency approximation. The main data processing was done for the accelerometer signals only.

The normalized short time Fourier transform (STFT or the spectrogram) of the pressure sensor data is shown in Figure 1. It can be seen that at the time interval from 35 s until the end of the test there is strong spectral component due to the rumble event in the frequency range $460-520 \mathrm{~Hz}$. The second and the third harmonics of this component are also present and can be found at the corresponding multiple frequencies, although the time interval where they can be observed is significantly shorter than that for the fundamental harmonic.

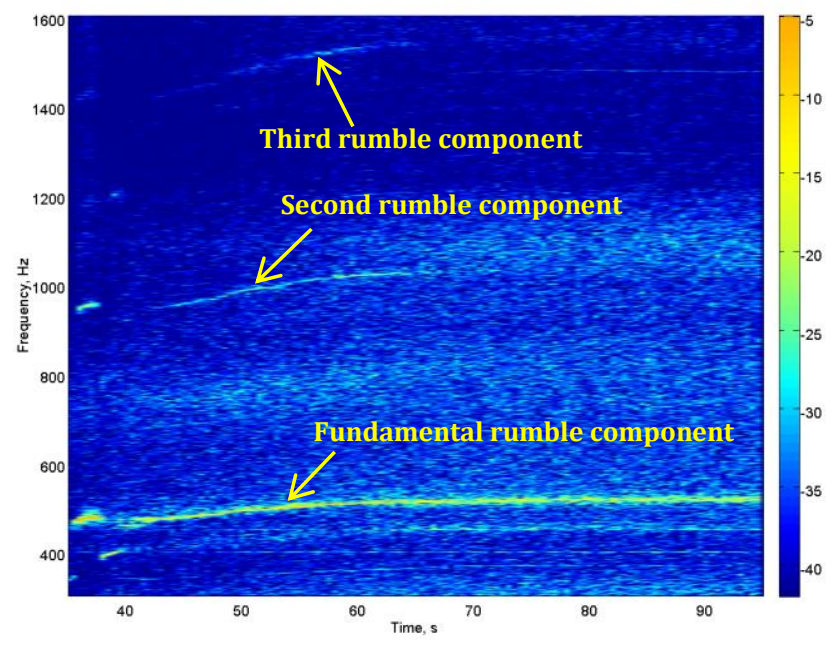

Figure 1. The normalized short time Fourier transform of the pressure sensor data with $\mathrm{Z}$-axis expressed in $\mathrm{dB}$ scale.
In the STFT of the casing accelerometer (Figure 2), the fundamental rumble component is also present, but the time range where the component is clearly visible is limited to 40 $\mathrm{s}-70 \mathrm{~s}$ interval. The second harmonic is not visible; the third harmonics can be observed in the time interval $45 \mathrm{~s}-60 \mathrm{~s}$.

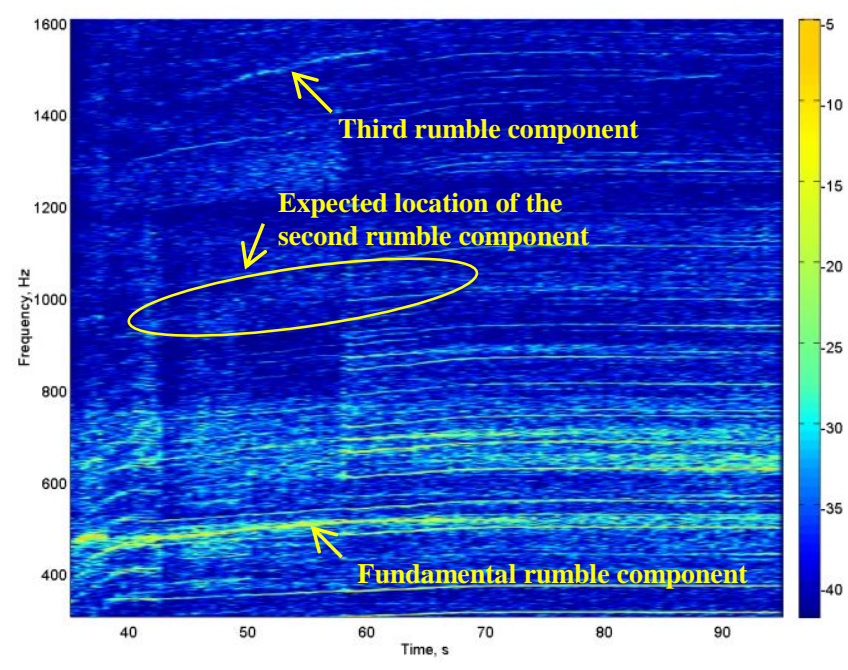

Figure 2. The normalized short time Fourier transform of the accelerometer data with $\mathrm{Z}$-axis expressed in $\mathrm{dB}$ scale.

Considering the STFT results for the pressure sensor and the accelerometer, and using the estimates of the shafts' speeds (from the corresponding tachometer signals) it was found that the frequency of the fundamental rumble component is not matching any of the integer or fractional shaft order frequencies in the whole time range of the event (Figure 3).

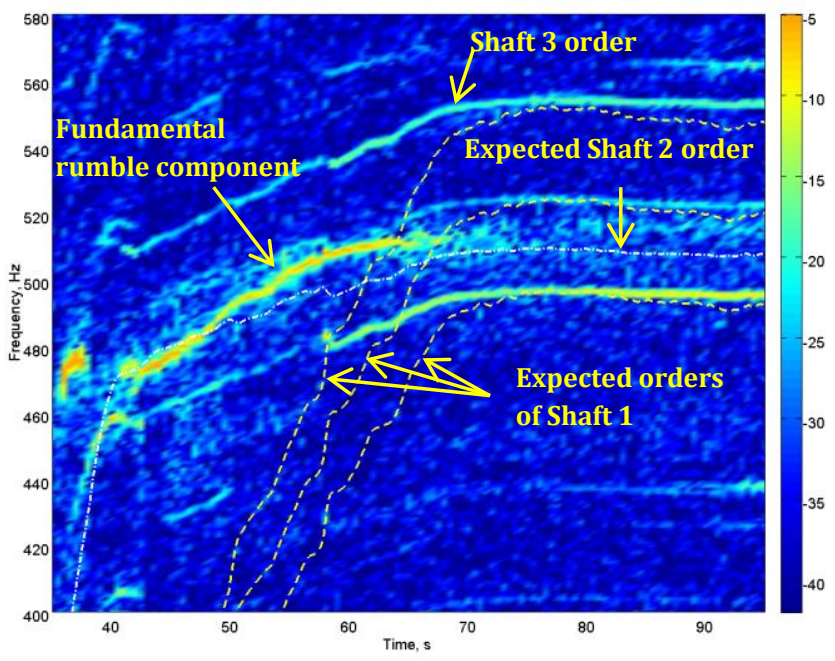

Figure 3. Selected area of the accelerometer data short time Fourier transform with fundamental rumble component and nearby shaft orders.

Therefore, the shaft speed, which can be estimated from the tachometer signal or from the vibration data itself, cannot be used for identification of the rumble position in time and 
frequency domains. In this work it is proposed to obtain the information about the frequency location of the rumble event from the STFT of the pressure sensor data. In the future, this procedure will be based on the accelerometer signal only.

\section{RESULTS AND DISCUSSION}

As three rumble components are visible in the STFT of the pressure data, the values for the bicoherence components at frequency locations $\left(f_{\text {rumble }}, f_{\text {rumble }}\right)$ and $\left(f_{\text {rumble }}, 2 f_{\text {rumble }}\right)$ can be considered, where $f_{\text {rumble }}$ is the fundamental frequency of the rumble event obtained by using the STFT of the pressure sensor data. During the data analysis it was found that the bicoherence component at frequency location $\left(f_{\text {rumble }}, 2 f_{\text {rumble }}\right)$ is less sensitive to the rumble presence in comparison to the component at $\left(f_{\text {rumble }}, f_{\text {rumble }}\right)$ for both pressure and acceleration data. Thus, the short time chirp Fourier bicoherence defined by Eq. (2) has been estimated at the frequencies $\left(f_{\text {rumble }}, f_{\text {rumble }}\right)$ using the data from the accelerometer. The external windows of $3 \mathrm{~s}$ length with $1 \mathrm{~s}$ step between them were used to obtain the dependency of the short time chirp Fourier bicoherence magnitude in time over the duration of the entire test data. Every external window was split into segments of $0.25 \mathrm{~s}$ each with $80 \%$ overlap between them. Hamming weighting window was applied to the segment data in order to reduce leakage. The estimates of the short time chirp Fourier bicoherence magnitude plotted against time are shown in Figure 4.

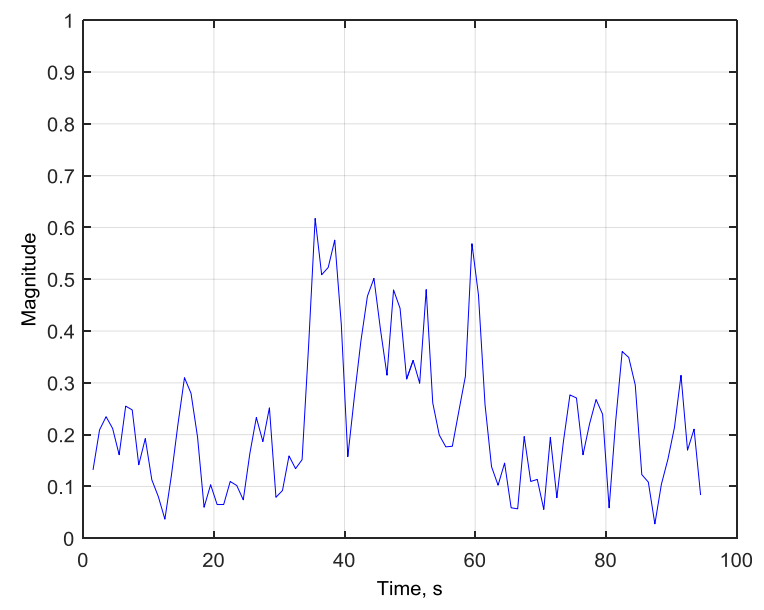

Figure 4. The short time chirp Fourier bicoherence.

It can be seen from the Figure 4 that the short time chirp Fourier bicoherence demonstrates increased values starting from approximately $35 \mathrm{~s}$ of the data until the $60 \mathrm{~s}$ of the data. This matches very well the observed behavior of the STFT for both pressure sensor and accelerometer, presented in Figure 1 and Figure 2.
Evaluation of the effectiveness of the short time chirp Fourier bicoherence for the rumble detection was performed based on comparison with the short time bicoherence defined by Eq. (1). For both techniques, manual one-off optimization of the main parameters was undertaken, based on the maximization of the chosen efficiency criterion, i.e. the Fisher criterion (Young \& Fu, 1986):

$$
F=\left(\mu_{2}-\mu_{1}\right)^{2} /\left(\sigma_{1}^{2}+\sigma_{2}^{2}\right)
$$

where $\mu_{1}, \mu_{2}, \sigma_{1}^{2}, \sigma_{2}^{2}$ are the mean values and variances for two groups of values being analyzed. The Fisher criterion in Eq. (3) characterizes numerically separation between the data within the area where the rumble is present and the data outside of this area.

The parameters optimized separately for both techniques are the external window size (starting from $2.5 \mathrm{~s}$ to $10 \mathrm{~s}$ with 0.5 $\mathrm{s}$ step) and the segment size (from $0.3 \mathrm{~s}$ to $1.2 \mathrm{~s}$ with $0.1 \mathrm{~s}$ step), while the overlap between segments and the time step between the consequent external windows were constant and equal to $80 \%$ and $1 \mathrm{~s}$ respectively. As the amount of the experimental data is limited it was decided to increase it by adding the small amount of random noise in a number of independent trials. Thus, for each parameter combination 100 repeat calculations were performed with addition of the Gaussian noise using the signal to noise ratio of $30 \mathrm{~dB}$.

The time interval for the rumble appearance was defined as 35 to $60 \mathrm{~s}$ based on the observations of the STFT of the pressure data. Respectively, the data prior to the $30^{\text {th }}$ second and after the $65^{\text {th }}$ second from the record beginning are considered as rumble-free.

The estimates of the short time Fourier bicoherence versus the time and the histograms of the bicoherence values with and without presence of the rumble are shown in Figure 5 and Figure 6 respectively.

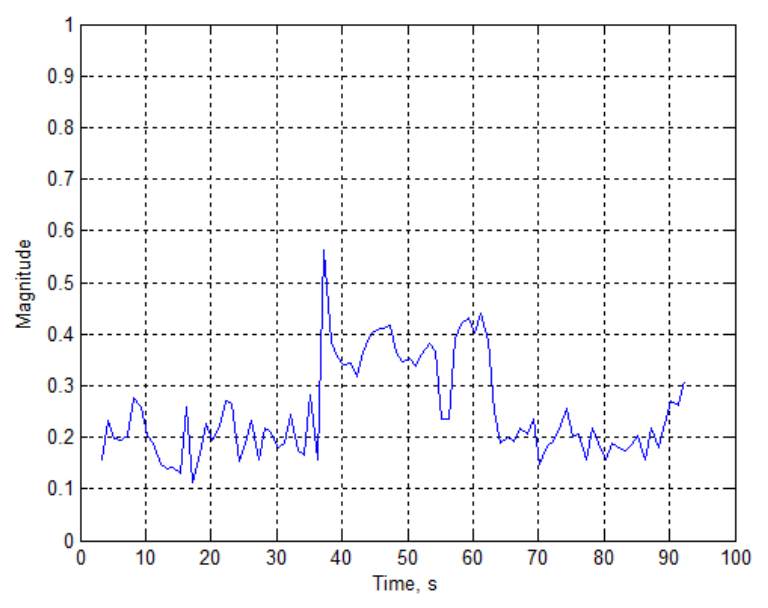

Figure 5. The estimates of the short time bicoherence based on Fourier transform.

In Figure 6, the histogram corresponding to the case when the rumble is present contains the bicoherence values from the 
interval 35 to $60 \mathrm{~s}$ as defined previously; another histogram contains all the bicoherence values outside this interval and corresponds to the case when the rumble is not present.

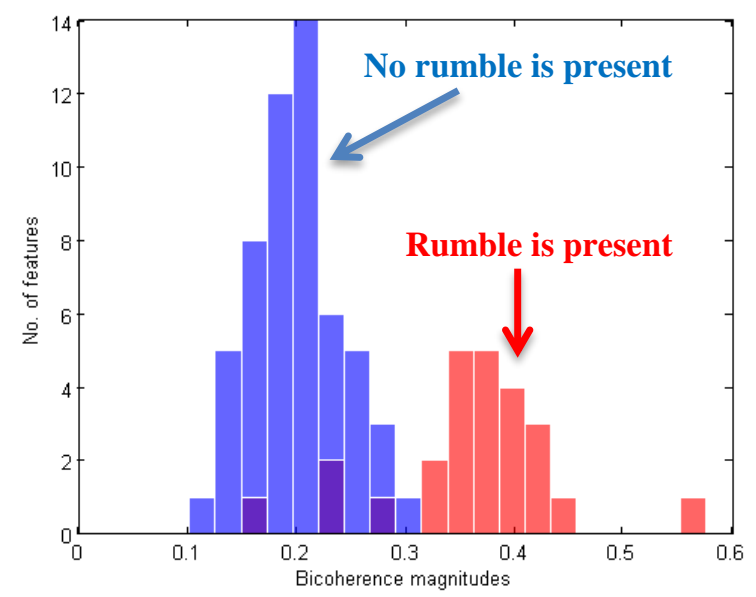

Figure 6. Histograms of the short time bicoherence based on Fourier transform using the data with and without rumble.

It can be seen that the bicoherence based on the Fourier transform is able to provide the separation between the case of normal operation of the turbine and the case when the rumble is present. An example of a solution for the rumble detection is a thresholding based on the magnitude values of the bicoherence values. For in-service detection of the rumble, the threshold can be set at the level of 0.3. It should be noted that due to the overlap between the groups of the bicoherence values characterizing the cases it is not possible to achieve $100 \%$ correct in-service rumble detection.

Figure 7 and Figure 8 show the estimates of the short time chirp Fourier bicoherence against the time and their histograms for cases of the turbine data with and without rumble presence respectively. Figure 7 demonstrates much clearer the rise of the short time chirp Fourier bicoherence values within the time interval corresponding to the rumble presence in comparison to the case when the short time Fourier bicoherence was used (Figure 5). It is confirmed by histograms in Figure 8, where there is no overlapping between groups of the chirp Fourier bicoherence data, meaning that using the threshold of 0.2 the error free inservice rumble detection can be potentially achieved.

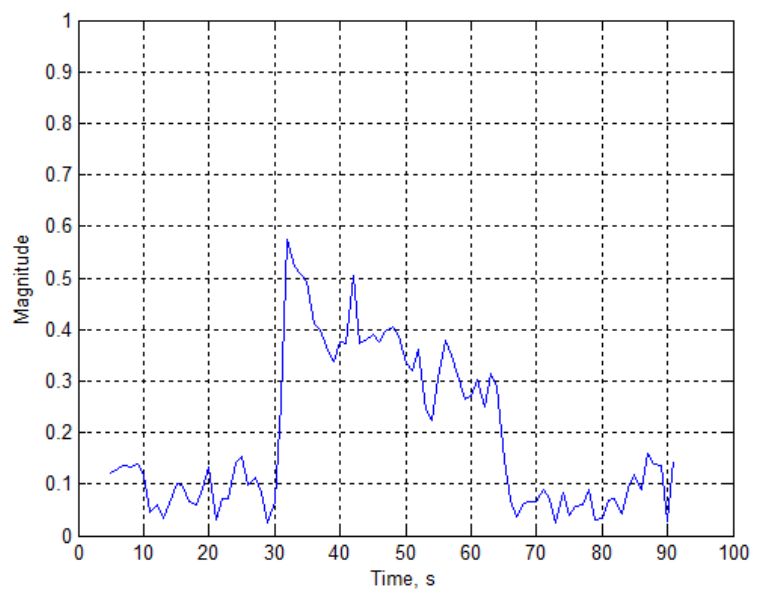

Figure 7. The short time chirp Fourier bicoherence estimates.

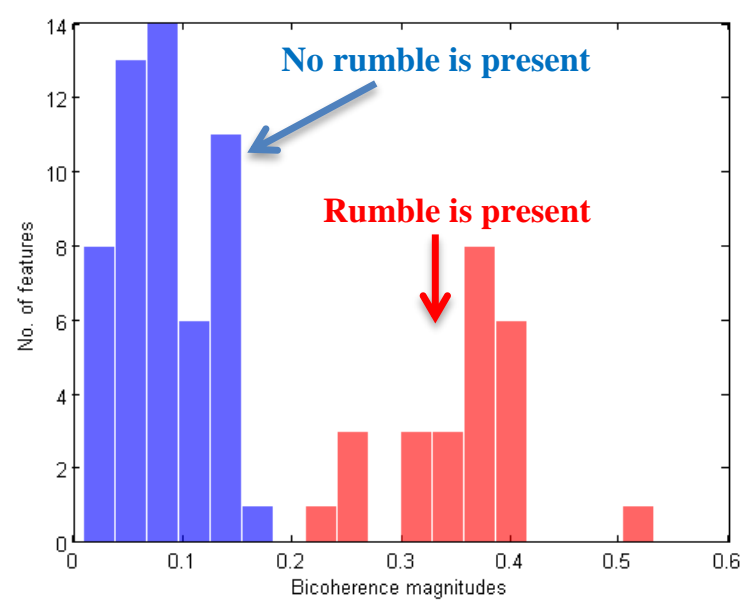

Figure 8. Histograms of the short time chirp Fourier bicoherence using the data with and without rumble.

At the present time, there is no strategy for establishing the threshold automatically. In the future, the threshold selection can be performed using the training data from multiple cases of rumble occurrence. The quantitative evaluation of results using the selected efficiency criterion confirmed that implementation of the short time chirp Fourier bicoherence for the rumble detection allows better detection of the rumble event comparing with the short time bicoherence based on the Fourier transform. Value of the Fisher criterion defined by Eq. (3) obtained for the chirp Fourier bicoherence was 14.1 against 3.8 for the bicoherence based on the Fourier transform. These results were achieved by using the optimal parameters for each technique: $10 \mathrm{~s}$ external window and 0.4 $\mathrm{s}$ segment size for the chirp Fourier bicoherence and $6.5 \mathrm{~s}$ 
external window and $0.3 \mathrm{~s}$ segment size for the bicoherence based on the Fourier transform.

The improvement in the detection efficiency when the short time chirp Fourier bicoherence is used instead of the bicoherence based on the Fourier transform can be explained by the adaptation of the chirp Fourier bicoherence to the nonstationary nature of the rumble phenomenon.

\section{Conclusions}

The short time higher order chirp Fourier bicoherence is applied for the first time for the transient rumble detection. The effectiveness of this technique is compared with the short time bicoherence based on the Fourier transform. It was found that the bicoherence based on the short time chirp Fourier transform allows for more effective rumble detection (the Fisher criterion value is 14.1) in comparison with the bicoherence based on the Fourier transform (the Fisher criterion value is 3.8) using the optimal signal processing parameters for both techniques.

The thresholding decision making technique applied to results from both techniques has shown the clear advantage of the bicoherence based on the short time chirp Fourier transform over the bicoherence based on the Fourier transform for in-service rumble detection. The error free detection of rumble can be achieved by proper selection of the detection threshold when the bicoherence based on the short time chirp Fourier transform is used.

Further improvement in the in-service rumble detection precision is expected in the cases when more advanced decision making techniques will be applied.

The implemented short time chirp Fourier bicoherence is an effective technique for in-service rumble detection because it is capable to suppress Gaussian noise, normalized and deal with signals with varying frequency of the rumble event

The work is expected to be continued in the future. It is planned to extend the current amount of data, used to demonstrate the efficiency of the technique, to a data library, containing multiple examples of combustion instabilities from different turbines. A detailed statistical analysis can be performed at that stage. The problems of the automated detection of rumble will be also considered.

\section{ACKNOWLEDGEMENT}

The authors thankfully acknowledge the financial support from Innovate UK/ EPSRC (UK) grant TP 101291.

\section{REFERENCES}

Combuster rumble. Patent EU EP 2199680A1, published June 23, 2010.

Grabill, P., Seale, J., Wroblewski, D. \& Brotherton, T. (2002). iTeds: the intelligent turbine engine diagnostic system. In Proc. of 48th International Instrumentation Symposium, May 5-9, San Diego, CA.
Wroblewski, D. \& Grabill, P. (2001). Analysis of gas turbine vibration signals for augmentor fault detection. In Proc. of the 37th AIAA/ASME/ASEE Joint Propulsion Conference and Exhibit, Salt Lake City, UT, 8-11 July.

Knock and rumble detector for internal combustion engines. Patent US 3540262, published Nov 17, 1970.

Gelman, L. \& Ottley, M. (2006). New processing techniques for transient signals with non-linear variation of the instantaneous frequency in time. Mechanical Systems and Signal Processing, Vol. 20, pp. 1254-1262.

Gelman, L. \& Petrunin, I. (2010). Time-frequency higher order spectra with adjustment to the instantaneous frequency variation, International Journal of Adaptive Control and Signal Processing, Vol. 24, No. 3, pp. 178187.

Gelman, L. \& Petrunin, I. (2007). The new multidimensional time/multi-frequency transform for higher order spectral analysis, Multidimensional Systems and Signal Processing, Vol. 18, No. 4, pp. 317-325.

Ebrahimi H. B. (2006). Overview of gas turbine augmentor design, operation and combustion oscillation, ILASS Americas, 19th Annual conference on liquid atomization and spray systems, Toronto, Canada, May.

Young, T. \& Fu, K-S. (1986). Handbook of pattern recognition and image processing. Academic Press: Orlando, FL.

Williams, F.A. (1985). Combustion theory: The fundamental theory of chemically reacting flow systems. Menlo Park, Calif: Benjamin/Cummings Pub. Co.

Richman, M.H., \& Richman, M.S. (2000). Active Combustion Control of Military Gas Turbine Engines. NATO Symposium on Active Control Technology for Enhanced Performance Operation Capabilities of Military Aircraft, Land Vehicles and Sea Vehicles, Braunshweig, Germany.

Yao, Z., Gao, Z., Zhu, M., Dowling, A.P. \& Bray, N.C. (2012). Combustion rumble prediction with integrated computational-fluid-dynamic/low-order-model methods. Journal of propulsion and power, 28(5), 10151025.

Lubarsky, E. \& Yeshayahou, L. (1998). Experimental investigation of flame holding system for the suppression of ramjet rumble. Twenty-Seventh Symposium (International) on Combustion / The Combustion Institute, 27(2), 2033-2037. doi:10.1016/S0082-0784(98)80049-0.

Dowling, A.P. \& Mahmoudi, Y. (2015). Combustion noise. Proceedings of the Combustion Institute, 35, 65-100. doi: 10.1016/j.proci.2014.08.016.

Hermann, J., Orthmann, A., Hoffman, S. \& Berenbrink P. (2000). Applications of active combustion control to Siemens heavy duty gas turbines. NATO RTO Meeting on Active Control Technology, Braunschweig, Germany.

Yu, K.H., Wilson, K.J. \& Schadow, K.C. (1998). Liquidfueled active instability suppression. Proc Combust Inst, 27, 2039-2046. 
Livebardon, T., Moreau, S., Gicquel, L., Poinsot, T. \& Bouty, E. (2016). Combining LES of combustion chamber and an actuator disk theory to predict combustion noise in a helicopter engine. Comb. Flame, 165, 272-287.

Motheau, E., Nicoud, F. \& Poinsot, T. (2014). Mixed acoustic-entropy combustion instabilities in gas turbines. Journal of Fluid Mechanics, 749, 542-576. doi: 10.1017/jfm.2014.245.

Hochgreb, S., Dennis, D., Ayranci, I., Bainbridge, W. \& Cant, S. (2013). Forced and self-excited instabilities from lean premixed, liquid-fuelled aeroengine injectors at high pressures and temperatures. Proceedings of
ASME Turbo Expo, Paper GT2013-95311, pp. V01BT04A023. doi:10.1115/GT2013-95311.

Gelman, L., Petrunin, I. \& Komoda, J. (2010). The new chirpWigner higher order spectra for transient signals with any known non-linear frequency variation. Mechanical Systems and Signal Processing, 24, 567-571. doi: 10.1016/j.ymssp.2009.07.004.

Gelman, L. \& Gould, J.D. (2007). Time-frequency chirpWigner transform for signals with any nonlinear polynomial time varying instantaneous frequency. Mechanical Systems and Signal Processing, 21, 29803002. doi: 10.106/j.ymssp.2007.05.003. 